First publ. in: Phase transitions in surface films [proceedings of the NATO Advanced Study Institute and International Course on Phase Transitions in Surface Films, held June 19 - 29, 1990, in Erice, Sicily, Italy], 1991, pp. 135-151

\title{
NEUTRON SCATTERING STUDIES OF QUANTUM FILMS
}

\author{
H.J.Lauter, H.Godfrin, V.L.P.Frank and P.Leiderer*
}

Institut Laue-Langevin, BP $156 \mathrm{X}, \mathrm{F}-38042$ Grenoble. France

: Institut fur Physik, Universitat Konstanz, D-7750 Konstanz,FRG

\section{INTRODUCTION}

The phase diagrams of a monolayer of adsorbed gases or light molecules on l?at substrates look similar to the phase diagrams in 3-dimensions (3-D). This means that the usual coexistence regions, the criple point and the critical point are present as well in 2 -dimensions (2-D) as in 3-D '. The phase diagrams can be studied by adsorption isotherm or heat capacity measurements, which reveal mainly the coexistence regions or the phase boundaries, respectively. However, the substrate can not always be regarded to be ideally flat. In many cases the adsorbate does see the adsorption sites of the substrate and locks into it commensurate phase (C-phase). In the case of graphite as substrate the $(\sqrt{3} \times \sqrt{3}) \mathrm{R} 30^{\circ}$ overstructure is seen in many cases. This structure is shown in fig. 1 . The heavier rare gases and the light molecules $\left(\mathrm{N}_{2}, \mathrm{CD}_{4}\right.$ ) extibit the $\mathrm{C}$-phase only if the lattice parameter of the dense plane in 3-D is close to the nearest neighbor distance in the $\mathrm{C}$-phase. This is the case because the nearest neighbor distance nearly does not change in the densest plane if the adjacent planes are taken off which means the dimensionality changes from 3-D to 2-D. The quantum gases however a!l show a $C$-phase in their 2-D phase diagram despite a much

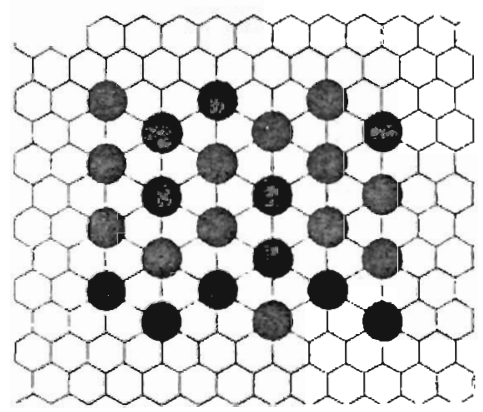

Fig. 1: Model of the commensurate $(\sqrt{3} \times$ $\sqrt{3}) \mathrm{R} 30^{\circ}$ overstructure on graphite.

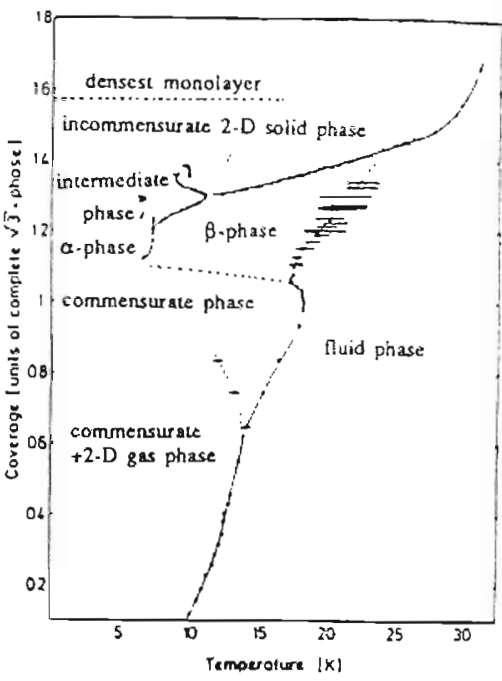

Fig. 2: Phase-diagram of D, on graphite [2] 
denser nearest neighbor distance in 3-D. This is due to the zero-point motion which gives a repulsive contribution to the nearest neighbor interaction and consequently a high compressibility to the system. Thus if as before the dimensionality is changed and many of nearest neighbor atoms are missing the 2-D lattice expands. If in addition the corrugation of the adsorption potential is added, the quantum gases recognize the dilute density structure of the C-phase as the ground state.

The phase diagram of $\mathrm{D}_{2}$ on graphite is shown in figure 2 as an example of an adsorbed quantum gas. The location of the phase boundaries has been determined by heat capacity measurements 2 . The definite allocation of the different phases to structures is given by scattering techniques. In this case it has been done by neutron diffraction and LEED 3,4 .

The inelastic neutron scattering gives additional information of the interaction between the adsorbed particles itself but also between the adsorbate and the substrate. In particular interesting is the search for the phonon gap at the zone center which characterizes the loss of the translational invariance of the adsorbed layer in the $\mathrm{C}$-phase. The transition from the Cphase to the incommensurate higher density phase is characterized by different intermediute phases as seen in figure 1 . Theories of the commensutrate-incommensurate transition predict domain walls in the transition region. In these domain wall phases the commensurate phase is still locally present. Thus the study of the phonon gap will reveal interesting features.

\section{EXPERIMENTAL}

The experimental set-up is described in Ref.5. The sample consists of a stack of exfoliated graphite sheets (Papyex) with a diameter of $2 \mathrm{~cm}$ and a height of about $7 \mathrm{~cm}$. The surface area is in the order of $200 \mathrm{~m}^{2}$. The graphite is a 2-D powder. Only the axis perpendicular to the basal planes of the graphite shows a certain order of a mosaicity of about $30^{\circ} \mathrm{FWHM}$. The coherence length of the adsorbed layer is about $300 \AA$ in the C-phase. The basal planes of the graphite are parallel to the scatiering plane of the neutron spectrometer. For the inelastic studies the IN3 spectrometer of the ILL has been used with a fixed final energy of $1 \mathrm{THz}$ and a Be-filter on the analyser end. The resolution across the elastic line was $0.03 \mathrm{THz}$. For the elastic studies ZYX-graphite was used with a coherence length of about $2000 \AA$.

The density of the adsorbate was controlled by adsorption isotherms. But also the highest intensity of the Bragg-peak of the adsorbate in the C-phase as a function of coverage at constant temperature determines the best commensurate phase $\rho=1 . \rho=1$ means that all adsorption sites in the $\mathrm{C}$-phase are occupied by adsorbed atoms or molecules. The definition of $\rho=1$ with diffraction is within $2-3 \%$ identical with the $\rho=1$ coverage defined by the highest melting temperature of the $\mathrm{C}$-phase in figure 2 . The small difference may be a real temperature effect.

\section{MEASUREMENTS}

\section{THE $(\sqrt{3} \times \sqrt{3})$ PHASE}

The verification of the $\mathrm{C}$-phase has to be done by diffraction. In fig. 3 neutron diffraction pattems are shown at various average adsorbate densities ${ }^{3}$. The spectrum $A$ at a slightly overfilled C-phase shows the maximum intensity at a momentum transfer $Q=1.702 \AA-1$ which corresponds for a triangular lattice to $4.26 \mathrm{~A}$, the nearest neighbor distance in the $C$ phase (fig. 1). All the spectra shown, the elastic and the inelastic ones, show difference counts. The signal from the cell and substarte without adsorbate has always been subtracted.

The inelastic neutron measurements have been taken at two different momentum transfers Q. The reason for that is depicted in fig. 4 which shows the reciprocal space of the triangular lattice of the $C$-phase. The scan taken with a $Q=1.7 \AA^{-1}$ collects all excitations with wave vector $q$ along the circle with radius $Q$ with the help of the Bragg-points which are marked by the vector $\tau$. These excitations with wave vector $q$ have mainly transverse polarization and the highest intensity is expected from the zone boundary phonons because of the high density of 
states. If a phonon gap exists at the zone center a second high density of states is expected at this point. As the 2-D powder averaging crosses also the $\Gamma^{-}$-points a second peak is expected in energy in the scan with a $Q=1.7 \AA^{-1}$.

The scan taken with $\mathrm{Q}=0.85 \AA^{-1}$ collects the longitudinal zone boundary phonons due to the 2-D powder averaging (fig.4). So with the correct choice of the momentum transfer different modes can be separated even in a powder-like sample.

The scans with the different momentum transfers are shown in figure 5 . The data points in fig.5a $\left(\mathrm{Q}=1.7 \AA^{-1}\right)$ show clearly a double peak. The one at lower energies represents the phonon gap, whereas the one at higher energies shows the collected transverse zone boundary phonons. The scan with a $Q=0.85 \AA^{-1}$ (fig.5b) shows only one peak which results from collected longitudinal zone boundary phonons. The fit to the data is a two

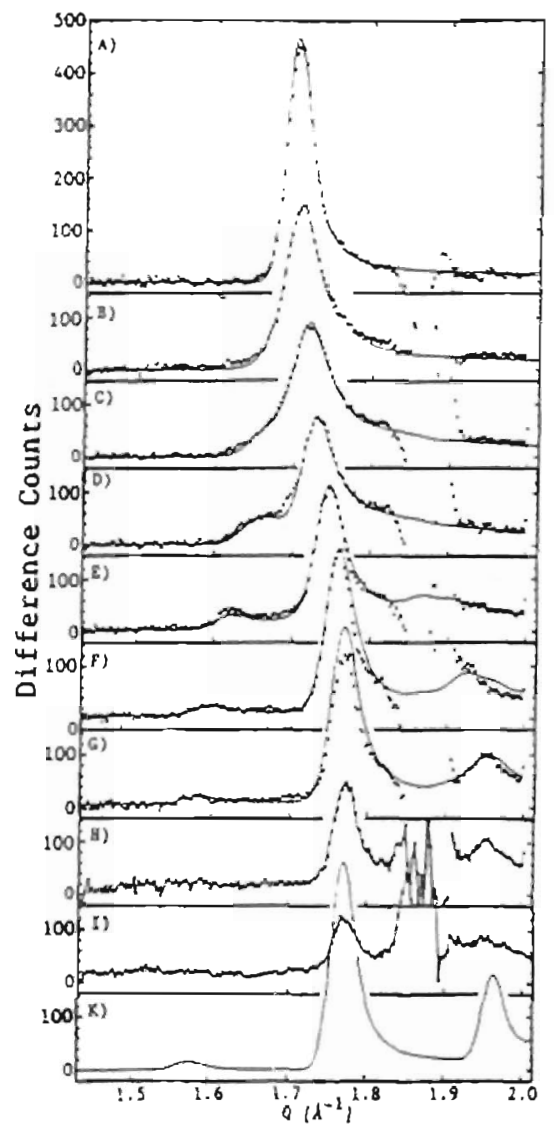

Fig.3: Diffraction pattern of $\mathrm{D}_{2}$ on graphite in the $C$-phase and $\alpha$-phase at various average densities of the adsorbate; A) $\rho=1.07$, B) $p=1.10$, C) $\rho=1.11$, D) $\rho=1.13, E) \rho=1.16$, F) $\rho=1.20, G) \rho=1.23, H) \rho=1.24,1) \rho=1.25 ; T=2 K$. The solid lines are fits with the model in fig. 7 [3]. 


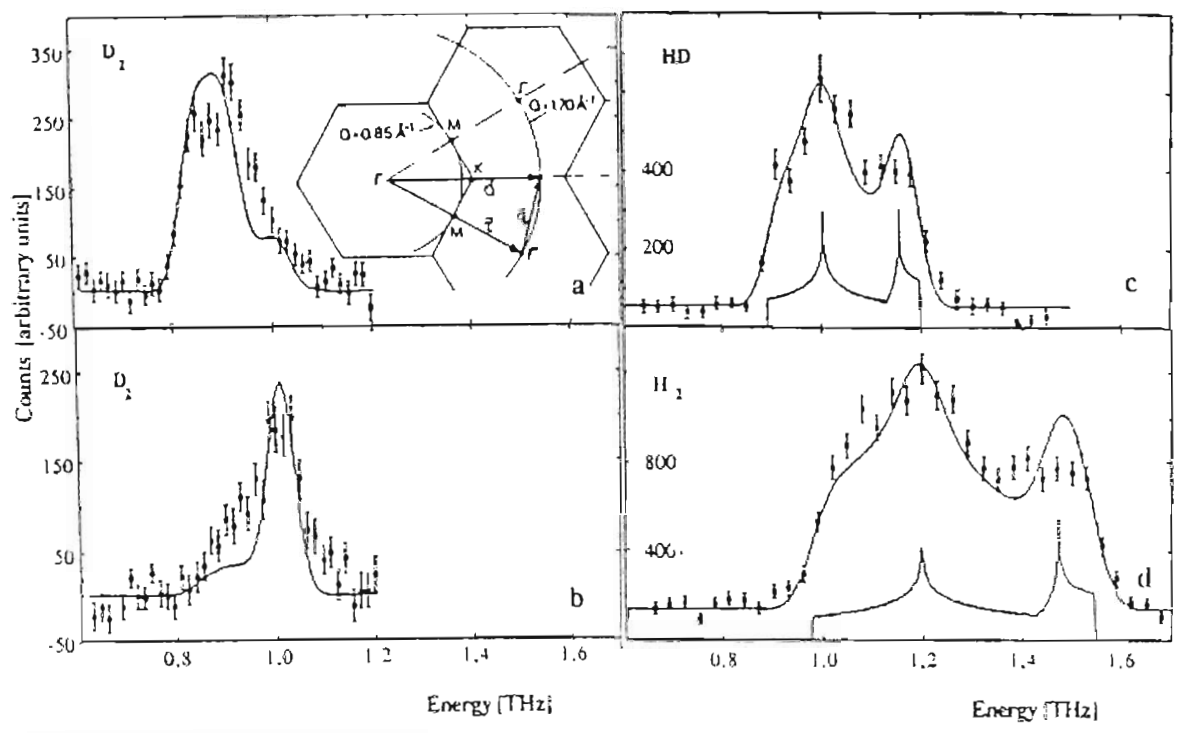

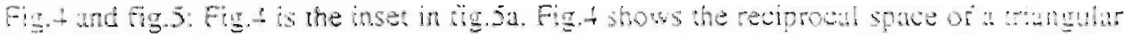
lattice. The circles are shown on which phonons are collected due 0 the powder weruging for $Q=1.7 \mathrm{~A}^{-1}$ and $\mathrm{Q}=0.85 \mathrm{~A}^{-1}$. $\mathrm{Q}$ is the cocal momentum transier: is a reciorocal latice vector and $q$ is the phonon wave vector [3]. Fig.5: Veutron inelastic data of the hydrogen isotopes adsorbed on graphite in the $C$-phase [8]. Q=0.35 $A^{-1}$ in b and $Q=1.7 A^{-1}$ in a.c and d. In a and $b$ che specra aken with $D_{2}$ are shown. which is a coneren scatterer. Wheress in $\therefore$ and $\mathrm{HD}$ and $\mathrm{H}_{2}$ is used. respectively, which are incoherent scamerers for nextons. So the densicv of stutes is measured.

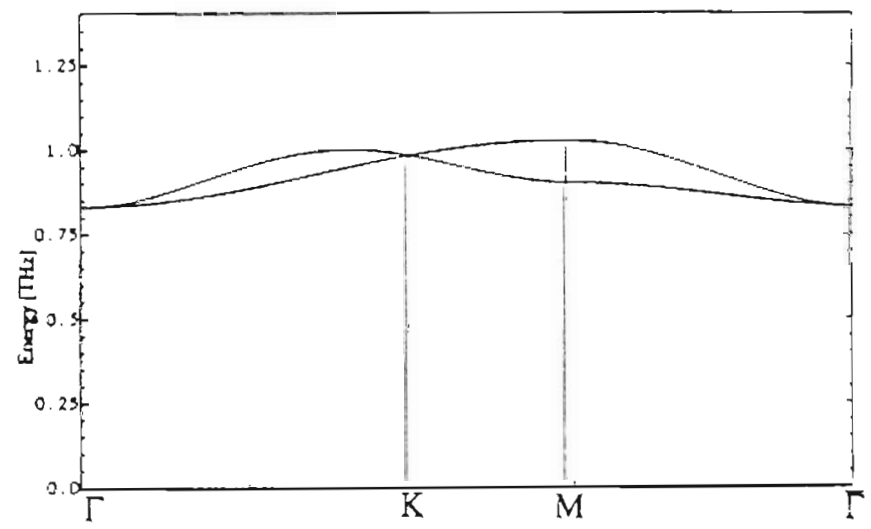

Fig.b: Calculated dispersion relation tor $\mathrm{D}_{2}$ on graprite in the $C \cdot$ phase $[5 ;$. 
parameter fit. The adsorbate molecules are thought 0 be connected by each other by a ne: of springs and each molecule is connected by a spring to the substrate ${ }^{5}$. The moctel is a very simple one and is not able to reproduce the clear separation between the phonon gap and the transverse zone boundary phonons seen in the data (fig.5a). But it is however interesting to note the difference in the spring constants. $\alpha$ is the spring constant between the adatoms and

Table 1: Parameters obained from the fits characterizing the density of states for the in-plane modes of the C-phase of the hydrogen atoms adsorbed on graphite. In parenthesis are the values of Ref.7. Indicated are the following: z.c.gap is the zone senter gap cnergy. widn is the width of the density of states, tanspetk and long peak are the peaks arsing from the transverse and longiudinal phonons in the density of sates, respectwely. All watues

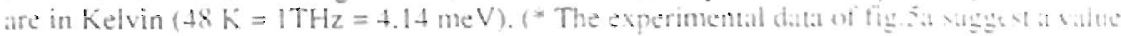
of $43.3 \mathrm{~K}$ for the trunverse perk)

\begin{tabular}{|lllll|}
\hline & $\mathrm{H}_{2}$ & $\mathrm{HD}$ & $\mathrm{D}_{2}$ \\
\hline \hline Z.c.gap & $47.3(46.6)$ & 43.2 & 40.0 & $(36.9)$ \\
\hline widh & $27.5(42.1)$ & 14.7 & 9.5 & $(14.8)$ \\
\hline (ranls.peak & $57.9(64.9)$ & 48.3 & $43.3 *(44.2)$ \\
\hline Iong.peak & $71.4(83.8)$ & 55.3 & $48.1(50.3)$ \\
\hline
\end{tabular}

Table 2: Parameters that characterize the adsornate graphite st wem th the commentinte

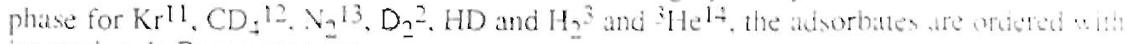
increasing de Boer parameter.

a The de Boer parameter indicates the yuantum character of the adsorbate.

b This column presents the width of the in-plane phonon densiry of states (DOSi. Thene

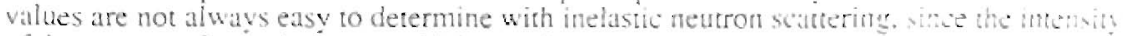
of the structure factor decreases with increasing energy.

c The phase diagrams of Kr, N2 and CD4 present a commensumte phase region that canth to higher temperatures, when the total coverage is slighly higher than the commensulc me: Details of the phase diagrams can be found in rets. 15, 16 and 17. respectuely.

\begin{tabular}{|c|c|c|c|c|c|c|c|c|}
\hline \multirow[t]{2}{*}{ Adsorbate } & \multirow{2}{*}{$\begin{array}{l}\text { Mass } \\
\text { |a.u.| }\end{array}$} & \multirow{2}{*}{$\begin{array}{l}\text { de Boer" } \\
\text { parameser }\end{array}$} & \multicolumn{2}{|c|}{ Lennard-Jones parameter } & \multirow{2}{*}{$\begin{array}{c}\text { Gap energy } \\
|K|\end{array}$} & \multirow{2}{*}{$\begin{array}{l}\text { Gap rano } \\
\text { Ameadseak }\end{array}$} & \multirow{2}{*}{$\begin{array}{c}\text { DOS uidth } \\
{[\mathrm{k}]}\end{array}$} & \multirow{2}{*}{$\begin{array}{c}\text { Melting } \\
\text { Temperature }\end{array}$} \\
\hline & & & $c|K|$ & $g(A)$ & & & & \\
\hline $\mathrm{Kr}$ & 838 & 0.10 & 1653 & 3.63 & 8.7 & 1.0 & 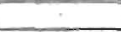 & -1250 \\
\hline $\mathrm{CD}_{4}$ & 200 & 0.23 & 137.0 & 3.68 & 745 & 0.9 & $\sqrt{x}$ & -58 \\
\hline $\mathrm{N}_{2}$ & 280 & 0.42 & 35.6 & 3.32 & 193 & 1.7 & 4 & -74 \\
\hline $\mathrm{b}_{2}$ & 40 & 1.26 & 35.2 & 2.95 & मय० & 1.1 & 95 & $1 \times 5$ \\
\hline HD & 30 & 1.43 & 35.9 & 2.95 & 432 & 1.1 & TS & 194 \\
\hline $\mathrm{H}_{2}$ & 2.0 & 1.74 & 36.7 & 2.95 & 47. & 1.11 & 25 & .205 \\
\hline 3 He & 30 & 3.10 & 101.2 & 2.56 & 104 & 0.1 & गा1 & .05 \\
\hline
\end{tabular}

$\beta$ the in-plane one between the adsorbate molecule and the substrate at the atiorpuon sitc. has been determined to $0.016 \mathrm{~N} / \mathrm{m}$ and $\beta$ to $0.182 \mathrm{~N} / \mathrm{m}$. The ratis is about $1 / 10$. This small ratio is equally well seen in the rather flat dispersion in fig.6. Thus the molecule exhibis nearly "Einstein behavior" 6 , but the influence of adatom-adatom interuction is still visible through the dispersion. Calculations ${ }^{7}$ are in good agreement with the value of the energy of the phonon gap, however the adatom-adatom interaction visible by the width of the density of states is still by a factor of 1.5 coo small (see table 1). This indicates that probubly not the parameters at the minimum of the interaction potential have to be modified but the shape of this potential at a larger intermolecular spacing due to the increased intemolecular spancin the C-phase. 
The effect of the isotope mass can be probed by using in addition to $\mathrm{D}_{2}$ also HD and $1 / 2$ as adsorbate 8 . The spectra are shown in fig. $5 \mathrm{c}$ and $5 \mathrm{~d}$. Both spectra make use of the incoherent scattering cross section of $\mathrm{HD}$ and $\mathrm{H}_{2}$. The calculated density of states is seen as a solid line in the lower part of the figures. The line through the points is the density of states folded with the resolution of the instrument. "The characterivic values of the dispersion relations are summarized in table 1 . The same model as described for $\mathrm{D}_{2}$ has been applied io the other isotopes. The theory ${ }^{7}$ describes well the isotope shift which is not only due to the different mass but also dure to the anharmonicity of the potentials. The isotope effect is seen with decreasing mass as well in the shift of the phonon gap to higher energies is in an increasing width of the density of states. As with $D_{2}$ the width of the density of states is wider by the same factor 1.5 (table 1).

The C-phase has also been detected for other adsorbates and a collection of gap energies and widths of the density of states is given in table 29,10 . The gap energy is related to the curvature of the corrugation of the in-plane adsorption potential at the adsorption site. The agreement between theorerical calculations of the gap energy and the measured values is indicated in the 7 th column of table $27,9,10,18$. The effective curvature is influenced by the movement of the atoms or molecules in the adsorption potential. All the quantum gases are governed by the zero point movement, so that the gap stays nearly unchanged up to the melting temperature of the $\mathrm{C}$-phase. $\mathrm{Kr}$ and $\mathrm{CD}_{4}$, however, show a lowering of the gap energy by a factor of 2 when the temperature is raised to the melting.temperature due to the enhanced mean-square displacement of the adsorbate and the anharmonicity of the adsorption potential $9.19,20$.

Also the width of the density of states exhibits the difference between the quantum gases and the heavier gases. The quantum gases show a smaller width of the density of states, because the nearest neigbor distance in the $\mathrm{C}$-phase is increased with respect to the one in 3-D. This leads to a lower interaction between adsorbate atoms. The heavier gases show more interaction, because the nearest neigbor distance in 3-D matches the one in 2-D. The high value of $3 \mathrm{He}$ should be taken with care. On one side the very high zero point motion may allow for a higher adsorbate-adsorbate interaction, but on the other side we are not absolutely sure about the interpretation of the data 14.

\section{THE COMMENSURATE-INCOMMENSURATE TRANSITION}

Once the inelastic signals are understood in the C-phase the adjacent phases can be investigated. This will be described first for the $\alpha$-phase. In fig. 3 several scans are shown taken at different coverages and constant temperature. They follow a path from the $\mathrm{C}$-phase into the $\alpha$-phase (fig.2). The diffraction peak shifts with increasing density to higher Qvalues according to the compression. At the same time satellites are moving outwards from the main peak. The feature around $1.88 \AA^{-1}$ is the (002) graphite peak, wrich is due to an interference phenomena. It is of no importance for this study and cuts unfortunately out a

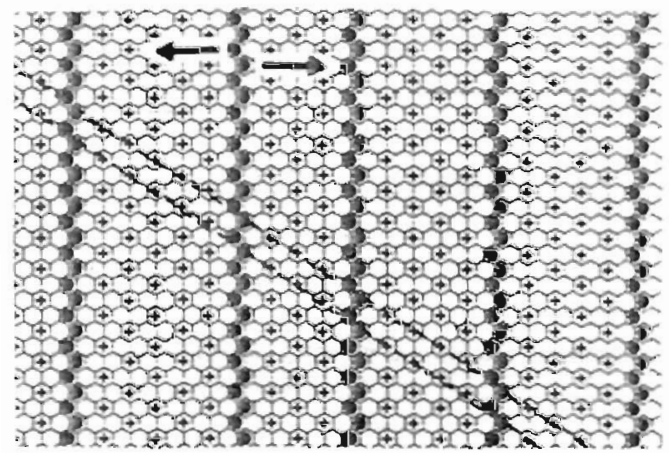

Fig.7: Striped superheavy domain wall model for $\mathrm{D}_{2}$ on graphite [3]. 


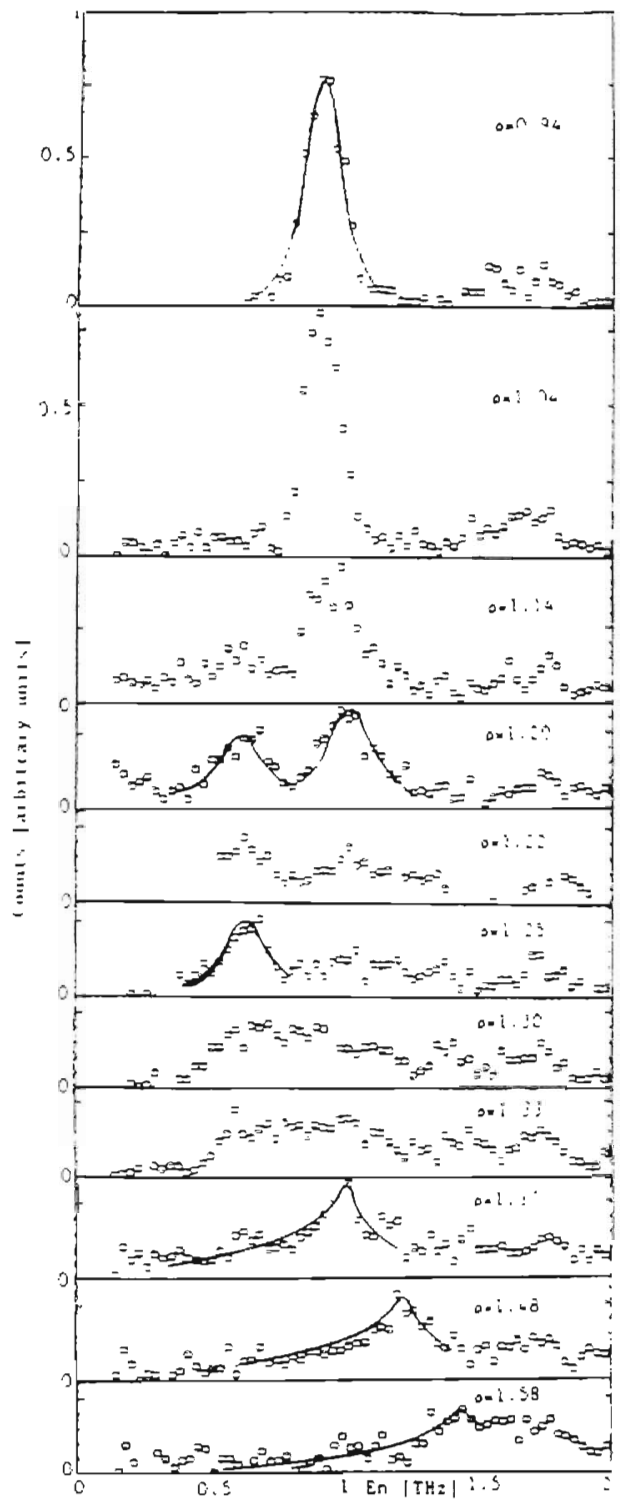

Fig.8: Neutron inciastic data of $D_{2}$ on. graphite at $T=4 K$ for various coverages $\left(Q=1.7 \AA^{-1}\right)$ (9). . Tie ines are guides to the eye. 


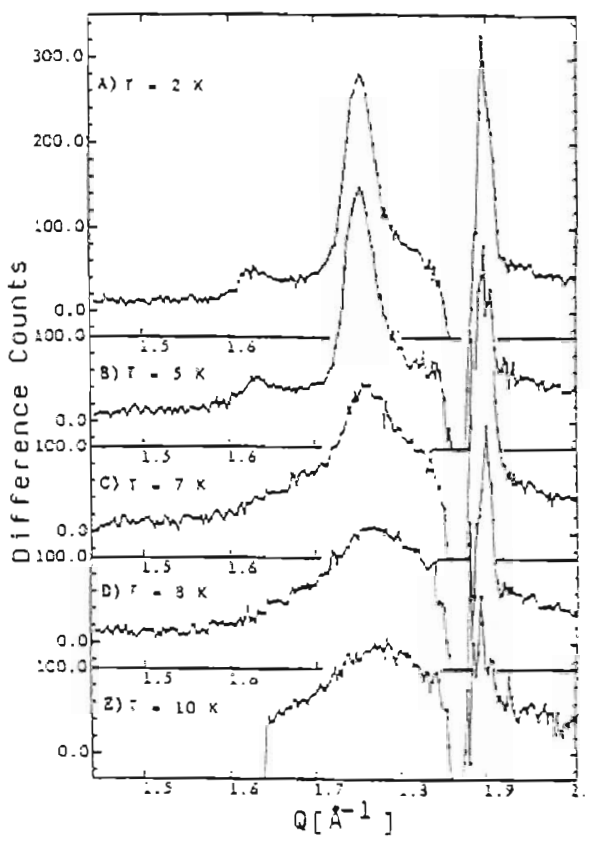

Fig.9: Diffraction pattern of $\mathrm{D}_{2}$ on graphite at constant coverage $p=1.16$ (see fig. 2) at various temperatures showing the $\alpha-\beta$ transition between $5 K$ and $7 K[3 b]$.

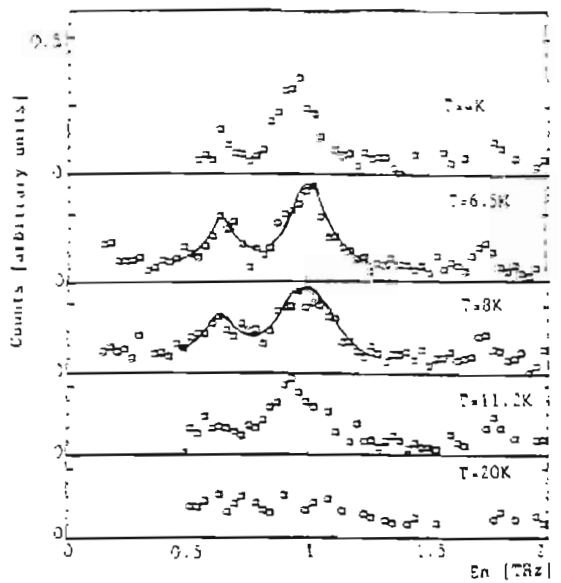

Fig. 10: Neutron inelastic data of $D_{2}$ on graphite at constant coverage $p=1.16$ (see fig.2) at various temperatures across the $\alpha-\beta$ transition [9]. The lines are guides to the eye. 
certain region in $Q$, where the signat of the adsorbate is not observable. The fit to the data has been done with a model of striped superheavy domain walls 3,4 which is depicted in fig.7. For a more detailed discussion the reader is referred to the Refs. 3 and 4 . It should only be mentioned that the crosses in fig. 7 represent the molecules still in the commensurate position. The filled circles mark the molecules in the domain walls in an ideal position. However they are too close and a certain relaxation indicated by the arrows takes place. In the applied model the distance between the domain walls has a distribution which depends on the domain wall density. The width of the domain walls is fairly siall. It extends only across 4 rows of molecules. This is again due to the high compressibilty of the 2-D quantum gases as mentioned in the introduction.

The inelastic studv of the domain wall suucture is sliown in fig.8 21. The top two specta are taken in the C-phase and show the phonon gap and the marsiverse zone boundary phonon in one peak due to the relaxed instrument resolution (0).06 $1 \mathrm{~Hz})$ used for these scans. II the averige density is further increased the $\alpha$-phase is entered (ke fig. 2 ) and the signal aromd I THz siarts to decrease. This is understood as due to the amoun of the molecules in the C. phatse decreasing due to the model of the domain walls. A the same lime a signal at athou $0.6 \mathrm{THz}$ appears which finally at $p=1.25$ is the on!y signal to be seen. This signil attributed 10 an exciation of the amain wats is still to he coulated. It is best at the densest $\alpha$-phatse $(\rho=1.25$, see fig.2). This is a first example ot the usafulness of the inelastic studies io complete and to understand the events in this phase itigram.

The following scans in fig. 8 show that in the region between $\rho=1.30$ and $p=1.35$ the signals can not be any more resolved probably due to 100 many different excitations in this phane which was modeled by a hexagonal heavy domain wall strucsure 3,4 . In the region beyond the density of $\rho=1.33$ the inelastic response changes again and indicates the pure transverse zore boundary phonon of an incommensurace 2-D solid.

Thi stexi object to study is the $\beta$-phase. In fig.9 diffraction patterns atre shown tiken at constant coverage $p=1.16$ as a function of temperacure 3 . At $T=2 \mathrm{~K}$ and $5 \mathrm{~K}$ the already

known salellite structure of the aphase is seen. which could be modeled by vinger superheavy domain walls. For higher temperatures than the $\alpha-\beta$ transition $(i j t-2)$ the specrum looks like a liquid structure factor in particular if the temperature is raised. The structure of this "reentrant liquid" was not known, but got new interest because this phase seems to be separated from the normal 2-D liquid by very broad peats in specitic has \{fig.2〉. The inelastic neutron measurements are shown in fig. 10. They have also been latien with the relaxed resolution (fig.8). So again the excitations of the commensurate parts (phonon gap and transverse zone boundary) are seen and at lower energy the signal from the domain walls. Here no change in the spectrum is seen if the temperaure crosses the u tansition at $7.2 \mathrm{~K}$. The consequence is that the domain walls do still exist in the $\beta$-phatse because the excitation belonging to them are still visible and also the excitations trom in commensurate phase. The solution for a stuctural picure is to introduce patches of domain walls as depicted in fig. 11 . This model still allows for the i: whitic featurs and the wucure factor seen as inser in fig. I 3 , fits the daca. This is a proof that the 3 -phase is a disorderedi domatn wall phase. The evolution with temperarure cian be madeled by shorter and sinorte: doman wal's until melting around 20K. Perhaps the unbindng of doman walls can be treated $i:$ the class of Kosterliz-Thouless transitions 22.23

The phase diagram of he monolaver of the heliums adsorbed on graphite atre similar lo the anes of the hydrogens in that a pronounced C-phase shows up with a transition to the incommensurate phase probably via a striped superheavy doman wall philse $2+26$. NG presen here the identification of the donain wall (D) phase of the as a striped superhes: domain wall pirase.

In fiug. 12 the explored coverage range berween 0.05 and 0.35 atomi $A$ - of it le on graphite is show 1s. The density of the adsorbed layer can be calculated from the diftaction peath position if a homogeneous triangular lattice is assumed. The first five points in figr.1 1 show :1!! the sime ordinate of $0.0636 \mathrm{at} / \AA^{2}$. the density of the C-phase. The abscissal s calibution (the cont number of adsorbed atoms on the suffice of the simple is siven by the amount of 


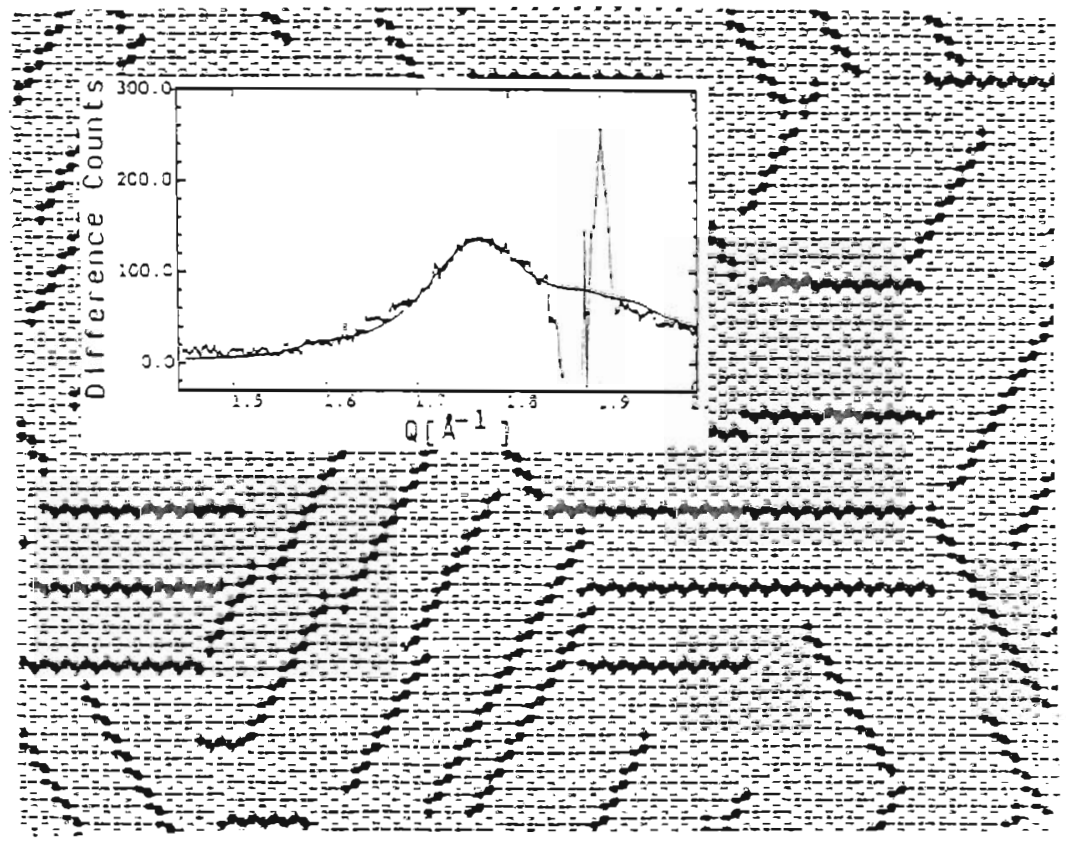

Fig. 11: Model for the $\beta$-phase. It is made up of parches of the $\alpha$-phase structure [ 30 ]. The inset shows an overlay of a measured spectrum in the $\beta$-phase (spectum $c$ in fig.9) and calculated one using the strucrure from the same figure [3b]. 
${ }^{3} \mathrm{He}$ that produces the highest Bragg-peak intensity within the $\mathrm{C}$-phase. This detmes the hest C-phase: all adsorprion sites of the C-phase on the graphite are occupied by an idsorbate arom. This calibration was first made with $\mathrm{D}_{2}$ on graphite ${ }^{3}$ and defined a value of $14.3+\mathrm{cc}$ (STP) of gas. This agrees with ${ }^{3} \mathrm{He}$, despite its low scattering power and high nentron absorption cross section.

The straight full line in fig. 12 through the best C-phase point and the origin does not matth the poins in the $[\mathrm{Cl}$-phase. This shows clearly that the calibration of the C-phase and of the incommensurate (ICI) phase do not coincide. One possible explanation is that the effective surfice area of the C-phase is smaller than the one of the ICl-phase. Anolher possibility may: be that in the ICI-phase atoms are additionally adsorbed on other planes than the basil ones. The calibration of the $\mathrm{ICl}$-phase is shown in fig. 12 by the dashed line, which does not past through the origin. The difference at monolayer completion anounts already to $6 \%$.

The deduced densities in the D-philee (fig. 2) show a behavior that is equivalent to the calse of D_ 3.4 . which has been identified as a striped superheavy domain wall phate. The mush las. tavorable scattering conditions of 3 He with respect to $\mathrm{D}_{2}$ made it impossible to mealsure amy saltellites. However. the chancterivic coverage dependence in 19.12 is a proot thall his doman wall phase exists as proposed by heat capacity measurements for $4 / 4 \mathrm{c} 25$. The density deduced from the diffraction perk position does of couse nor represen any more the real density of the layer, but serves just for visualization. The denest sage is renched whun

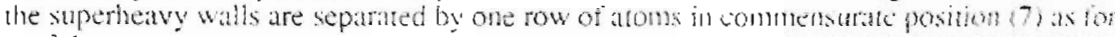
$D_{2}, i .1$.

\section{THE SECOND ADSOR!3ED LAYER}

For the helium isotopes, the second adsorbed layer is less dense than the first one -7 in contrast to the hydrogen isotopes 28 , where both layers have the whe demsity. The wethe: adsorption potential and the enhanced zero poin motion of the helum atoms are clewly visible in the low density of the second layer. Under the pressure of the second layer the density of the first layer imcreases shghty and finully locks into as so and $y * 0$ ) werstrume for ${ }^{3}$ He and ${ }^{4}$ He in respecively.

The second layer promotion is evidenced as a sharp knee betucen the $1 \mathrm{Cl}$ and $1 \mathrm{C}-\mathrm{p}$ hiw: in fig. 12. The increasing pressure of the second layer on the first one mduces a furber compression of the tirst laver. seen by the small slope in the IC2-phase. This slope increases slighty towards the S-phase. changing over to a zero slope at $0.2 \mathrm{at} / \mathrm{A}^{2}$. At this poin the densily of the first layer $\left(0.1106, \mathrm{~V}^{2} \mathcal{A}^{2}\right)$ corresponds 10 an $8 \$ 8$ overstmelure with rexpect the graphite, having 37 aroms per unit cell. The approtch to this clatr lock-in is marked in the S-phase by some diffraction peaks, whose line shape is betuer described by a double

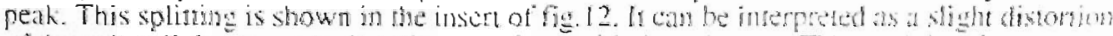
of the unit cell due to a one directional registry wh the substrate. This pumal regisury ateres for wo points with the $4 * 4$ lattice spaing and for one point with the 8 s one before firmtly the pure $8 * 8$ oversmucture is reached.

The diffraction of the second layer itsell cond be seen only at higher coneriges around a

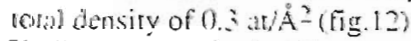

Similar features in the bilayer region are seen for the on graphite in 71 . 13 bo . For intensiy. reasons the signal from the C-phase could not be seen. Thus only the peak positions from the $\mathrm{ICl}$-phase (see fig.12) appear at low densities. For the same reason we coukt not calibrat the $x$-axis on the best $C$-phase as was done in fig. 12. Thus the $x$-axis is in sumare .ool of the amoun of the filling. The substrate used is Papvex with a coherence length of abou $350 \AA$. But in addition the data obtained with $\mathrm{ZYX}$-substrate coherence lagh ibou $2(x)() \mathrm{A})$ are shown in fig. 13 with a rescaling at the monolayer completion for the $\backslash-a x i$.

Again the second layer promotion is evidenced as a sharp knee al a denvity of $\left(\right.$ ). 112 al/ $\hat{A}^{2}$ ( ${ }^{3}$ He at ().106 at: $\mathrm{t}^{2}$ in fig. 12) and the increasing pressure of the second huer induces a small furcher compression of the first layer At a filling of $19 \mathrm{cc}^{3 / 2}$ the first liver locks ino an 


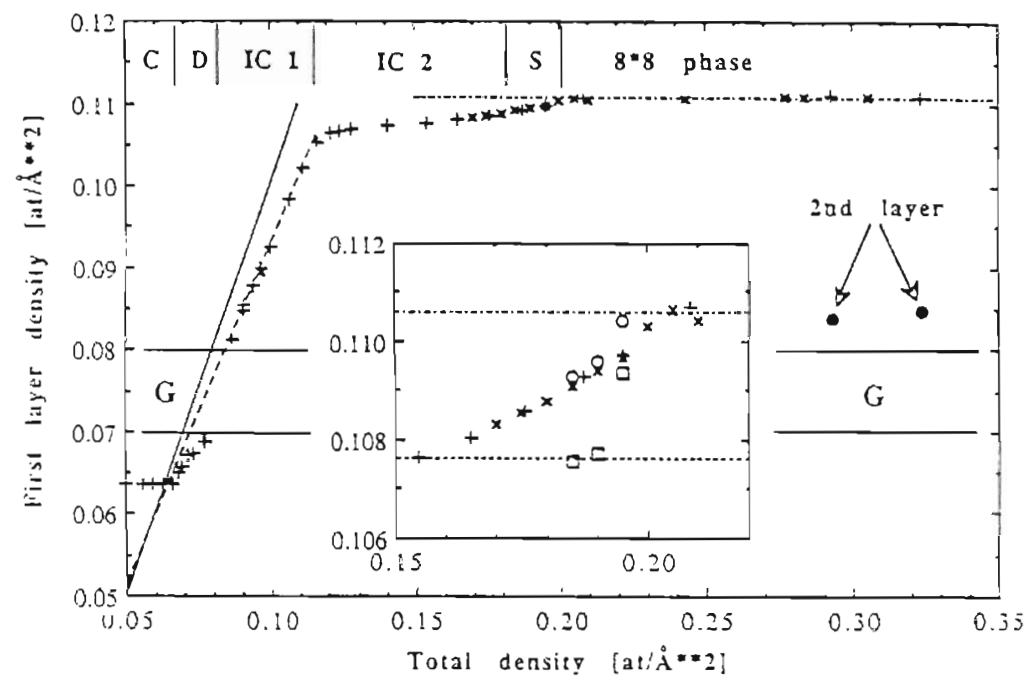

Fig. 12: Measured density of the first ${ }^{3} \mathrm{He}$ layer vs. total coverage at $\mathrm{T}=1 . \mathrm{OK}(+)$ and al $T=() .(96 \mathrm{~K}(\mathrm{x})$. (J) and $(0)$ indicate the splitting of the diftraction peak in the msert. $\bullet \cdot$ in the position of the second laver density. The identiticution of the diferent phats is dexcrilxd in the text. ( marks at region disturbed by at graphite background rellevtom. The full and lished line depict the monolayer behavior. The dashed-docted line shows the densily of the (5:8 phase. The dished line (insert) indicates the $4: 4$ density:

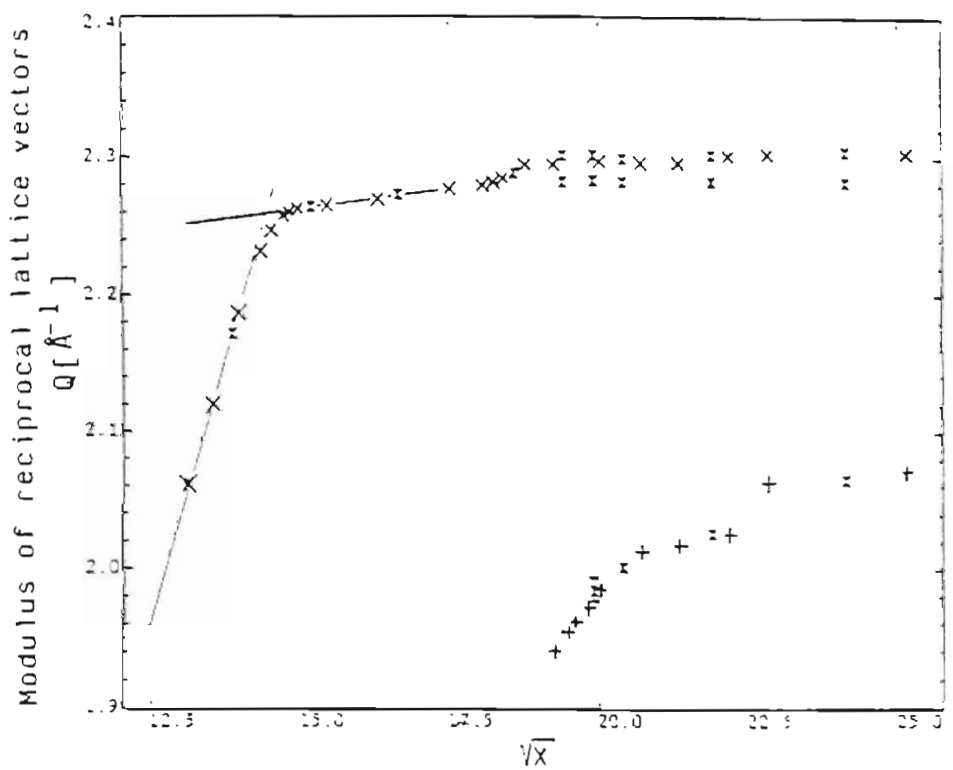

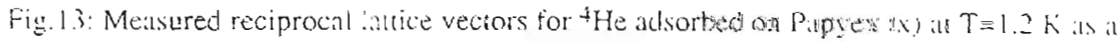
function of the square root of the total coverage taken in units of $\mathrm{cm}^{3} \operatorname{STP}(\mathrm{X}) .(x)$ and $(+)$ are the ZYX dara, rescales: to the coverage at the monolayer completion for the Papyex sinnple. 
oversm:cture with respect to the graphite. This time it is the $9 * 9$ overstricture for the $Z Y X$. subsirate. For the Papyex a splitring of the diffraction peak is observed, which indicates a one directional regisuy with the substrate induced by the small coherence length of this substrate.

The second layer diffraction peaks becone visible at a filling of $19 \mathrm{cc}^{3 / 2}$ and a compression of the second layer density is seen with the third layer promotion in fig. 13.30

\section{THE ${ }^{4}$ IE FILM}

A H He film on graphite is composed of iwo solid layers adjacent to the subsitate (below 2K) and subsequent liquid layers (see e.g.Ref.13). So the liquid ${ }^{4} \mathrm{He}$ film has two boundaries,

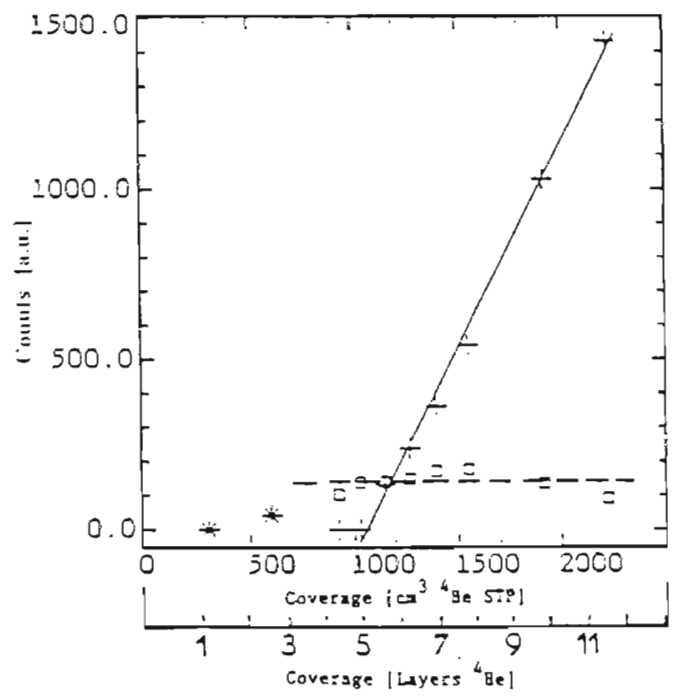

Fig. : : Intensities of the bulk wignal (+) and the interface signal (o) at the cnergy of 0.6 meV as a function of coverage ( ${ }^{4} \mathrm{He}$ on graphite powder 31 ). The signal at a coverage of a monoliyer and at 2.6 layers is marked by (*) (it is a constant background !); " $\mathrm{l}=(1) .8 \mathrm{~K}$.

the solid-liquid one and the liquid-gas one. Excitations can propagate along these intertices; which are the freezing-meiting wave ${ }^{29}$ and the ripplon ${ }^{30}$, respectively. Any exciation with a dispersion like a freezing wave cosld not yet be measured with neutron sciltering. But in: addition to the signals arising from the bulk ${ }^{4} \mathrm{He}$ some modes could be cietecied which have :o dispersion (the energy dues not change as a function with wave vector). These modes (at $0.4 \mathrm{meV}, 0.6 \mathrm{meV} . .$. ) are localized at the solid-liquid interface because they scill exist : the sample cell is completely filled with helium (this means that the liquid-gas interface is supressed) ${ }^{31}$. On the oiher hand these modes disappear between a coverage of 2.5 and 4 
layers as shown in fig. 14. This range has unfortunately not been investigated in more detail. But for coverages beyond 4 the intensity of this mode does not increase as function of coverage in agreement with the explanation that it is bound at the solid-liquid interface. These modes can be taken to explain the high transmission of phonons through the interface between a soilid and liquid helium (Kapitza-resistance). In contrast the bulk signal increnses linearly with coverage. It extrapolates to zero at a coverage of 5 layers. This indicates that in addition to the two solid layers three liquid layers do not contribute to superfluidity, if superfluidity is connected to the measurability of the phonon-roton dispersion curve. This statement will be refined for the $4 \mathrm{He}$ film on Payex-graphite, which is described in the following.

At the second interfacc a ripplon should be visible. Indeed ic could be meastured $31-33$ and stows the dispersion expected from theory 30 . The atribution of this mode to be a ripplon was strengthened by the fact that this mode disappears if the liquid-gas intertace is suppressed by filling the sample cell completely with helium. This is visualized in the figs. 15 and 16. In the ivures the different colors indicate the behavior of the intensity as a function of energy and momentum transfer. It is clearly seen in fig. 15 that besides the intensity on the phonon-roton curve there is incensity on an energetically lower lying branch. This branch coincides with the calculated dispersion of the ripplon using the parameter set in Ref.20. The agreement is very good, it seems to be so even up to $1.5 \mathrm{~A}-1$. At still higher $\mathrm{Q}$ the roton intensity combined with the one of the flat modes becomes to high to distinguish the ripplon signat. This good agreement allows 10 say that the temperature dependence of the surface tension is really based on an experimentally verified dispersion relation. It still rentains to prove the modified parameter set 20 by a theory.

In tig. 16 the result o: the completely filled sample cell is shown. In the region where the ripplon should show l:p, the colors are the same as in fig. 15. Only near the phonon-roton intensity and the flat bar due to the multiple scattering 31.32 the attribution of the colors to intensity has been modified. Thus this figure shows that no signal of the ripplon intensity is visible in fig. 16 the filled cell data, although below $0.7 \AA^{-1}$ it would have been distinguishable from the overwhelming quasi bulk phonon roton intensity. This disappearance proves that the ripplon signal is really bound to the gas-liquid intertace.

The fig. 17 shows the evolution of the signal height of the ripplon will corerage. Again the saturation shows that this mode is bound to an interface. It becomes visible above 3 lavers (two of them solid !). Like in fig. 14 the evolution of the bulk signal is exhibited. The extmpolation is made only with coverages below 5 layers. This shows that the bulk signal disappears at about 3.5 layers. There is no discrepancy with the dependence in fig. 14 because here in fig. 17 the scale is much finer and in principle a linear tail of the bulk signal height as a function of coverage is seen. (A rough exarapolation from still higher coverages extrapolates to about 4.5 layers in agreements with fig. 14.

In conclusion it has been shown that a lot of information can be drawn from adsorbates even on powder like graphite substrates using elastic and inelastic neutron scattering. These studies give insight into the dynamical behavior of the adsorbates in the commensurate phase itself, but which is also very valuable for the modelling of 2-D phases in the demonsirated case of the commensurate-incommensurate transition. All the adsorbed quantum gases have a connensarate-incommensurate transition, where the striped superheavy domain wall phase appears as intermediate phase. This domain wall phase shows again phase transitions ats a function of temperature which implies the creation of shorter pieces of domalin walls. The evolution of the first layer strucure is demonstrated towards the densest monolayer and under the pressure of the second layer. Due to the second layer pressure the first layer of the helium: show overstructures with the stostrate. In particular interesting are the interface excitations of an ${ }^{4} \mathrm{He}$ film. Ac the solid-licind hulium incurface localized excitations conld be detected and at the liquid-gas helian interface the ripplon could be measured. Thus an overview has been given what can be measured with neutron scattering in the calse of adsorbed quantum gases on graphite rareng from a submonolayer to a film. 

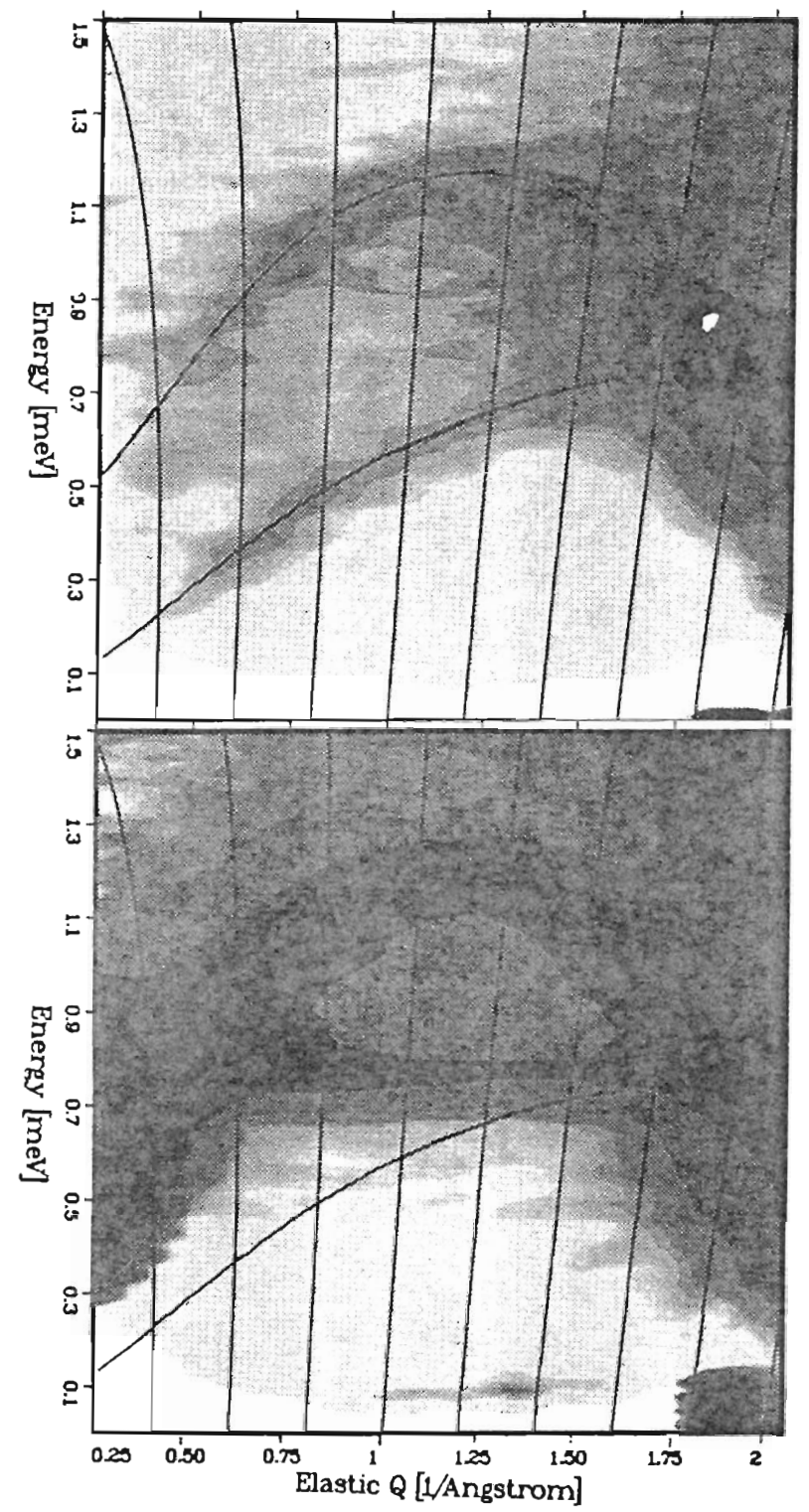

Fig. 15 (top)and 16 (bottom): Intensity along the phonon-roton curve and along the ripplon curve in thi energy-Q plane. The intensities originately displayed as different colors are visualized by the graduation from white to black, which marks the highest intensity (e.g. at the roton minimum in fig.15). Fig. 15 shows the signal from 5.06 adsorbed layers on Papyex and $11 \mathrm{~g} .16$ the signal from the completely with ${ }^{4} \mathrm{He}$ filled sample cell. In both figures the phonon-roton curve of bulk helium is seen as a black line as well as the ripplon dispersion. 


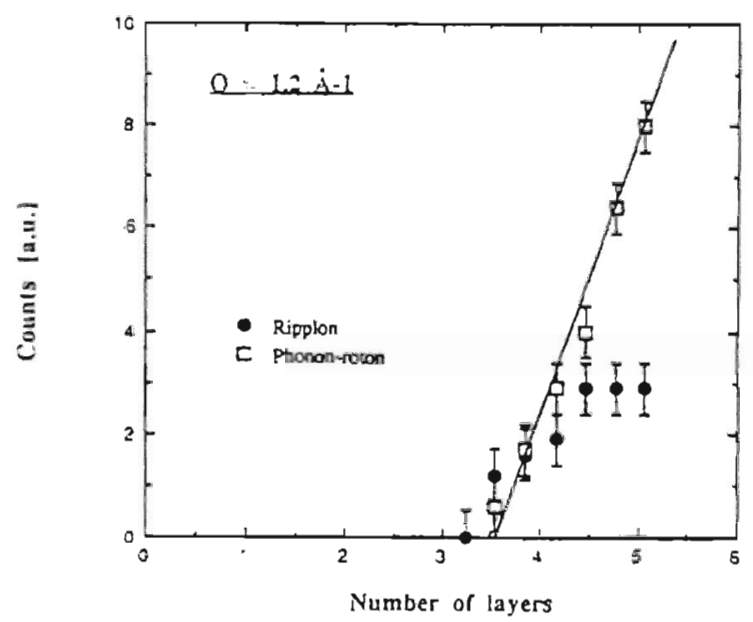

Fig.17: Intensities of the bulk signal (o) and the ripplon $(\bullet)$ as a function of coverage $\left({ }^{4} \mathrm{He}\right.$ on Papyex-graphite); $\mathrm{T}=0.6 \mathrm{~K}$.

\section{ACKNOWLEDGEMENTS}

This work has been partially supported by the Federal Ministry of Research and Technology (BMFT), F.R.G.

\section{REFERENCES}

1. Thomy A., Duval X. and Regnier J., Surf.Sci.Rep.1, 1 (1981)

2. Freimuth H. and Wiechert H., Surf.Sci. 178, 716 (1986)

3a.Schildberg H.P., Lauter H.J., Freimuth H., Wiechert H. and Haensel, Jap.J. Appl. Phys.26, 345 (1987), (Proc. 18th Int.Conf.on Low Temperature Physics, Kyoto);

3b.Schildberg H.P., Thesis, University of Kiel R.F.A. (1988)

3c. H.J.Lauter, in "Phonons 89", Hunklinger. S., Ludwig W. and Weiss G.edts.(World Scientific, 1990) p.871

4. Cui.J, Fain S.C., Freimuth H., Wiechert H., Schidberg H.P. and Lauter H.J., Phys.Rev.Len.60, 1848 (1987)

5. Frank V.L.P., Lauter H.J. and Leiderer P., Phys.Rev.Lett.61, 436 (1988)

6. Nielsen M., McTague J.P. and Passell L, Phase Transitions in Surface Films, Plenum Press (1980) p.127

7. Novaco A.D., Phys.Rev.Let.60, 2058 (1988)

8. Lauter H.J., Frank V.L.P., Leiderer P. and Wiechert H., PhysicaBl56\&157, 280 (1989)

9. Lauter H.J., Frank V.L.P., Taub H. and Leiderer P.,LT-19, PhysicaB 165\&166, 611 (1990)

Frank V.L.P., Lauter H.J., Godfrin H. and Leiderer P., NATO Workshop, Exeter 1990

10 Bruch L.W. same NATO Course

11 Frank V.L.P., Lauter H.J. and Taub H., unpublished data

12 Moeller T., Lauter H.J., Frank V.L.P. and Leiderer P., "Phonons 89", Hunklinger. S., Ludwig W. and Weiss G.edts.(World Scientific, Singapore 1990) p.919

13 Hansen F.Y., Frank V.L.P., Taub H., Bruch L.W., Lauter H.J. and Dennison J.R., Phys.Rev.Lett.64, 764 (1990)

14 Frank V.L.P., Lauter H.J., Godfrin H. and Leiderer P.,"Phonons 89", Hunklinger. S., Ludwig W, and Weiss G.edts.(World Scientific, Singapore 1990) p.1001 
15 Butler D.M., Litzinger J.A. and Steward G.A., Phys.Rev.Lett.44, 466 (1980)

16 Chan M.H.W., Migone A.D., Miner K.D. and Li Z.R., Phys.Rev.B.30, 2681 (1984)

17 Kim H.K., Zhang Q.M.and Chan M.H.W., Phys.Rev.B 34, 4699 (1986)

18 Ni X.Z. and Bruch L.W., Phys Rev.B 33, 4584 (1986)

19 Hakim T.M., Glyde H.R. and Chui S.T., Phys.Rev.B 37, 974 (1988)

20 Frank V.L.P., Lauter H.J., Godfrin H. and Leiderer P.,"Phonons 89", (World Scientific, Singapore 1990) p.913

21.Frank V.L.P, Lauter H.J. and Leiderer P., Jap.J.Appl.Phys.26, 347 (1987), (Proc. 18th Int.Conf.on Low Temperature Physics, Kyoto)

22. Kosterlitz M. and Thouless D.L., Prog in Low Temp.Phys. VII B, 371 (1987)

23.Halpin-Healy T. and Kardar M., Phys.Rev. B 34, 318 (1986)

24.Hering S.V, Van Sciver S.W and Vilchés O.E., J.Low Temp.Phys. 25, 793 (1976)

25.Motteler F.C, Thesis (Univ. of Washington, 1985)

26.Lauter H.J., Godfrin H., Frank V.L.P, and Schildberg H.P., LT-19, Physica B] $65 \& 166,597(1990)$

27.Lauter H.J.,Schildberg H.P., Godfrin H., Wiechert H.and Haensel R., Can.J.Phys. 65. 1435 (1987)

28.H.P.Schildberg, H.J.Lauter, H.Freimuth, H.Wiechert and R.Haensel, Jpn.J.Appl. Phys. 26, 343 (1987)

29.Keshishev K.O., Parshin A.Ya. and Babkin A.B., Sov.Phys. JETP 53, 362 (1981)

30.Edwards D.O. and Saam W.F., Prog. in Low Temp.Phys. VII A, 283 (1978)

31.Lauter H.J., Frank V.L.P., Godfrin H. and Leiderer P., Elementary Excitations in Quantum Fluids, Ohbayashi K and Watabe M. Eds.,Springer Series in Solid-State Sciences 79 (1989) p.99

Lauter H.J., Godfrin H. and Wiechert H., in "Phonon Physics",Kollar J.et. al. edss.. (World Scientific, Singapore 1985) p.842

32.Godfrin H., Frank V.L.P., Lauter H.J. and Leiderer P.,"Phonons 89", Hunklinger. S.. Ludwig W. and Weiss G.edts.(World Scientific. Singapore 1990) p.904

33.Lauter H.J., Godfrin H., Frank V.L.P. and Leiderer P., NATO Workshop, Exerer 1990 